\title{
Single-Valued Double Many-Body Expansion Potential Energy Surface of Ground-State $\mathrm{SO}_{2}$
}

\author{
S. P. J. Rodrigues, J. A. Sabín, and A. J. C. Varandas* \\ Departamento de Química, Universidade de Coimbra, 3004-545 Coimbra, Portugal
}

Received: September 12, 2001; In Final Form: October 30, 2001

\begin{abstract}
An accurate single-valued double many-body expansion potential energy surface has been obtained for the ground electronic state of the sulfur dioxide molecule $\left(\mathrm{SO}_{2}\right)$ by fitting novel ab initio energies suitably corrected by scaling its correlation energy. The stationary points of the new surface have been exhaustively analyzed, and the quality of the fit was appreciated from the stratified root-mean-square deviations between the points and the analytical potential.
\end{abstract}

\section{Introduction}

The sulfur dioxide molecule $\left(\mathrm{SO}_{2}\right)$ is an important pollutant whose role in the combustion of sulfur-containing materials ${ }^{1}$ and atmospheric photochemistry, as well as in atmospheric dynamics, is ubiquitous. Because of its importance, this molecule has been the subject of much experimental ${ }^{2-10}$ and theoretical ${ }^{11-15}$ spectroscopic work, in addition to studies of its predissociation mechanism. ${ }^{16,17}$ It also provides an important prototype for investigating vibration-rotation interaction and the onset of classical chaos, ${ }^{18-21}$ as well as normal-to-local mode transitions and quantum stochasticity. ${ }^{11,13}$

A large number of ab initio investigations on the ground and excited states of sulfur dioxide have appeared in the literature. ${ }^{16,22-30}$ Although there are some analytical potential energy surfaces for the $\mathrm{SO}_{2}$ ground state that are primarily based on spectroscopic data ${ }^{31-35}$ (the most recent is that of ref 15 which appeared during the writing of this work), it seems that attempts to obtain a global potential energy surface for this system from ab initio data are scarce. Such attempts include the many-body expansion fit to CASSCF energies of Lendvay et al. ${ }^{36}$ using a basis set of double- $\zeta$ quality and unpublished calculations and local fits. ${ }^{20,21,37,38}$

Concerning the features of the $\mathrm{SO}_{2}$, it is well established ${ }^{22,26}$ that like $\mathrm{O}_{3}$ the adiabatic single-valued ground-state potential energy surface of $\mathrm{SO}_{2}$ has four minima. Despite this similarity, ozone has three equivalent $C_{2 v}$ minima, whereas sulfur dioxide has a global minimum that corresponds to a bent $C_{2 v}$ OSO structure and to two $C_{s}$ minima. These minima are high in energy and correspond to superoxide structures of the type SOO, all of which belong to the same $\left({ }^{1} A^{\prime},{ }^{1} A_{1}\right)$ surface. The fourth minimum, which is the analogue of the cyclic isomer of $\mathrm{O}_{3}$, corresponds to a ring structure with $C_{2 v}$ symmetry, where the $\mathrm{O}-\mathrm{O}$ distance is smaller than the $\mathrm{S}-\mathrm{O}$ distances. ${ }^{22,26}$ This minimum belongs to another $\left({ }^{1} A^{\prime},{ }^{1} A_{1}\right)$ surface that crosses the previous one. As far as we know, attempts to locate the exact locus of this crossing have not been made, and this topic is beyond the scope of the present work.

The present contribution is organized as follows. In section 2 , we describe the ab initio calculations that have been carried out in the present work. The double many-body expansion (DMBE) formalism is discussed in section 3, where the details of the three-body dispersion, electrostatic, and extended Hartree-Fock (EHF) energies are reported in subsections 3.1., 3.2,

* Corresponding author. E-mail: varandas@qtvs1.qui.uc.pt. and 3.3, respectively. The main topographical features of the DMBE potential energy surface are analyzed in section 4. In section 5, we present the major conclusions.

\section{Ab Initio Calculations}

The ab initio energies have been calculated at the CASPT $2^{39}$ (complete active space second-order perturbation theory) level using a FVCAS (full-valence CAS) reference wave function (FVCAS/PT2) and the aug-cc-pVTZ Dunning basis set ${ }^{40,41}$ These calculations were carried out using the MOLPRO ${ }^{42}$ package. All calculations have been done using $C_{s}$ symmetry, and the bulk of the calculated ab initio energy points have been obtained for $\mathrm{O}-\mathrm{SO}$ Jacobi coordinate geometries in the region defined by $2.506 \leq R_{\mathrm{SO}} / a_{0} \leq 3.306,2.0 \leq r_{\mathrm{O}-\mathrm{SO}} / a_{0} \leq 6.0$, and $0 \leq \gamma /$ deg $\leq 180$. Except where mentioned otherwise, all quantitities will be reported in atomic units: $E_{\mathrm{h}}=4.359748$ $\mathrm{aJ}, a_{0}=5.29177 \times 10^{-11} \mathrm{~m}$. In addition, some extra points have been calculated in the vicinity of the local minima and for selected $\mathrm{S}-\mathrm{O}_{2}$ geometries. Because of convergence problems near the crossing points of the two ${ }^{1} A^{\prime}$ surfaces and the expected loss of quality of the CASPT 2 method in such crossing regions, ${ }^{39}$ some of the calculated points were not very accurate; hence, they were discarded. Therefore, from the initial ca. 650 geometries scanned, we have retained 587 ab initio points to use in the fit. The potential energy curves of $\mathrm{SO}$ and $\mathrm{O}_{2}$ have also been studied at the same level of theory ( 73 points in all).

The ab initio energies have been scaled using the double many-body expansion-scaled external correlation (DMBE-SEC) method, ${ }^{43}$ which is expected to provide an accurate semiempirical correction to the dynamical correlation calculated using MRCI or CASPT2 methods. The total interaction energy in the DMBE-SEC method is written as ${ }^{43}$

$$
V(\mathbf{R})=V_{\mathrm{FVCAS}}(\mathbf{R})+V_{\mathrm{DMBE}-\mathrm{SEC}}(\mathbf{R})
$$

where the FVCAS and DMBE-SEC components are defined as follows:

$$
\begin{array}{r}
V_{\mathrm{FVCAS}}(\mathbf{R})=\sum_{i=1}^{3} V_{\mathrm{FVCAS}}^{(2)}\left(R_{i}\right)+V_{\mathrm{FVCAS}}^{(3)}\left(R_{1}, R_{2}, R_{3}\right) \\
V_{\mathrm{DMBE}-\mathrm{SEC}}(\mathbf{R})=\sum_{i=1}^{3} V_{\mathrm{DMBE}-\mathrm{SEC}}^{(2)}\left(R_{i}\right)+V_{\mathrm{DMBE}-\mathrm{SEC}}^{(3)}\left(R_{1}, R_{2}, R_{3}\right)
\end{array}
$$

\subsection{1/jp013482p CCC: $\$ 22.00$ (C) 2002 American Chemical Society}

Published on Web 12/20/2001 


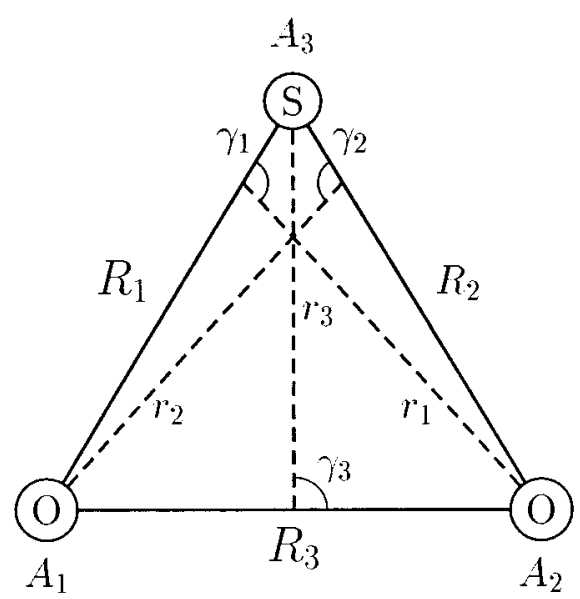

Figure 1. Summary of the coordinates used in the present work.

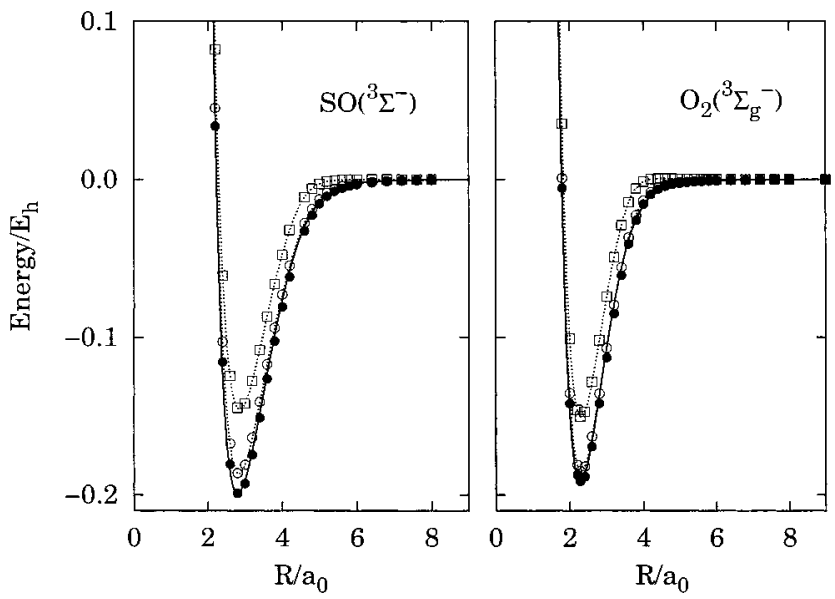

Figure 2. Calculated and scaled diatomic energies for $\mathrm{SO}$ and $\mathrm{O}_{2}$ : $\square$, FVCAS; O, FVCAS/PT2; - DMBE-SEC. Cubic spline interpolation of the ab initio energies are represented by the dotted lines while the corresponding EHFACE2U potential energy curves are shown by the solid lines.

The summations extend as usual to all diatomic $i \equiv \mathrm{AB}$ fragments. Moreover, $\mathbf{R}$ represents the set of all internuclear distances that define the molecular configuration space: $R_{1}=$ $R_{\mathrm{SO}(1)}, R_{2}=R_{\mathrm{SO}(2)}$, and $R_{3}=R_{\mathrm{OO}}$, (see Figure 1). In turn, the DMBE-SEC energies assume the following forms:

$$
\begin{gathered}
V_{\mathrm{DMBE}-\mathrm{SEC}}^{(2)}\left(R_{i}\right)=\left[V_{\mathrm{CASPT} 2}^{(2)}\left(R_{i}\right)-V_{\mathrm{FVCAS}}^{(2)}\left(R_{i}\right)\right] / F_{i}^{(2)} \\
V_{\mathrm{DMBE}-\mathrm{SEC}}^{(3)}\left(R_{1}, R_{2}, R_{3}\right)= \\
{\left[V_{\mathrm{CASPT} 2}^{(3)}\left(R_{1}, R_{2}, R_{3}\right)-V_{\mathrm{FVCAS}}^{(3)}\left(R_{1}, R_{2}, R_{3}\right)\right] / F^{(3)}}
\end{gathered}
$$

The scaling parameters $F_{i}^{(2)}$ and $F^{(3)}$ have been chosen from the requirement that the corrected ab initio diatomic curves reproduce the experimental dissociation energies ${ }^{44-46}$ and from the condition that the entire DMBE-SEC surface reproduces the well depth of the triatomic as taken from the experimental value reviewed elsewhere, ${ }^{28}$ respectively. The calculated scaling parameters are $F_{\mathrm{SO}}^{(2)}=0.7654, F_{\mathrm{O}_{2}}^{(2)}=0.8436$, and $F_{\mathrm{SO}_{2}}^{(3)}=1.331$. Figure 2 shows the calculated and scaled energies for the diatomic fragments, in addition to the fitted analytical EHFACE2U ${ }^{44}$ (extended Hartree-Fock approximate correlation energy including the united-atom limit of the diatomic) potential curves that will be discussed in the following section.
TABLE 1: Numerical Parameters of the EHFACE2U Potential Energy Curve for $\mathrm{O}_{2}{ }^{45,49}$

\begin{tabular}{lllc}
\hline$D / E_{\mathrm{h}}$ & 0.3946368556 & $R_{\mathrm{M}} / a_{0}$ & 2.2818 \\
$a_{1} / a_{0}{ }^{-1}$ & 2.6728294111 & $R_{0} / a_{0}$ & 5.6617 \\
$a_{2} / a_{0}{ }^{-2}$ & 1.7286687044 & $C_{6} / E_{\mathrm{h}} a_{0}{ }^{6}$ & 18.06 \\
$a_{3} / a_{0}{ }^{-3}$ & 1.1945319698 & $C_{8} / E_{\mathrm{h}} a_{0}{ }^{8}$ & 260.76896 \\
$\gamma_{0} / a_{0}{ }^{-1}$ & 2.1706286025 & $C_{10} / E_{\mathrm{h}} a_{0}{ }^{10}$ & 4932.4802 \\
$\gamma_{1}$ & 1.82153289295 & $\tilde{A} / E_{\mathrm{h}}$ & -6.4392 \\
$\gamma_{2} / a_{0}{ }^{-1}$ & 0.10883857476 & $\tilde{a}_{1} / a_{0}{ }^{-1}$ & -0.321468505
\end{tabular}

\section{DMBE Potential Energy Surface}

According to the $\mathrm{DMBE}^{47,48}$ method, the potential energy surface for $\mathrm{SO}_{2}$ assumes the form

$$
V(\mathbf{R})=\sum_{i=1}^{3} V_{i}^{(2)}\left(R_{i}\right)+V_{\mathrm{EHF}}^{(3)}(\mathbf{R})+V_{\mathrm{dc}}^{(3)}(\mathbf{R})+V_{\mathrm{elec}}^{(3)}(\mathbf{R})
$$

where $V_{i}^{(2)}, V_{\mathrm{EHF}}^{(3)}, V_{\mathrm{dc}}^{(3)}$, and $V_{\mathrm{elec}}^{(3)}$ represent the two-body energy terms, the three-body extended Hartree-Fock (EHF) energy, the three-body dynamical correlation energy, and the three-body electrostatic energy, respectively. To model the two-body energy curves, we have employed EHFACE2U ${ }^{44}$ potential energy curves. We refer the reader to the original papers for details about $\mathrm{SO}^{46}$ and $\mathrm{O}_{2}{ }^{45}$. (Because a misprint occurred in the latter, we reproduce in Table 1 the corrected parameters of the $\mathrm{O}_{2}$ potential $^{49}$ ).

3.1. Three-Body Dynamical Correlation Energy. The threebody dynamical correlation energy assumes the usual form of a summation in inverse powers of the fragment separation distances: ${ }^{50}$

$$
V_{\mathrm{dc}}^{(3)}=\sum_{i=1}^{3} \sum_{n} f_{i}(\mathbf{R}) \chi_{n}\left(r_{i}\right) C_{n}^{(i)}\left(R_{i}, \gamma_{i}\right) r_{i}^{-n}
$$

The first summation includes all atom-diatom interactions ( $i$ $\equiv \mathrm{A}-\mathrm{BC}) . R_{i}$ is the diatomic internuclear distance, $r_{i}$ is the separation between atom $\mathrm{A}$ and a certain point located in the $\mathrm{BC}$ diatomic internuclear coordinate, and $\gamma_{i}$ is the angle between these two vectors (see Figure 1). In turn, $C_{n}^{(i)}\left(R_{i}, \gamma_{i}\right)$ represent the atom-diatom long-range dispersion coefficients ${ }^{51}$ that will be defined later, $\chi_{n}\left(r_{i}\right)$ is a dispersion damping function, ${ }^{52}$ and $f_{i}(\mathbf{R})$ is a three-body switching function of the form ${ }^{50}$

$$
f_{i}=\frac{1}{2}\left\{1-\tanh \left[\xi\left(\eta s_{i}-s_{j}-s_{k}\right)\right]\right\}
$$

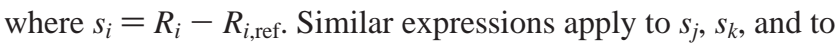
the other channels. The parameters have been chosen according to the criteria discussed in ref 50: $R_{1, \text { ref }}=R_{2 \text {, ref }}=2.74 a_{0}$; $R_{3 \text {,ref }}=4.31 a_{0} ; \xi=0.60 a_{0}^{-1}$; and $\eta=3$. As discussed elsewhere 46,50 an overestimation of the dynamical correlation due to eq 7 must be corrected by multiplying the two-body dynamical correlation energy terms for the $i$ th pair by $f_{i}(\mathbf{R})$, which has also been considered in the present work. It should be stressed that the choice of the point where the coordinates $r_{i}$ and $R_{i}$ are in contact is necessary for the definition of the coordinate transformations $\left(R_{1}, R_{2}, R_{3}\right) \leftrightarrow\left(R_{i}, r_{i}, \gamma_{i}\right)$. In the present work, we have chosen such a point to be the center of mass of the diatomic species (Jacobi coordinates). Of course, such a definition introduces the parameter $A_{i}$, which has values numerically identical to the those of the nuclei masses. These parameters will remain invariant for all isotopomers of $\mathrm{SO}_{2}$ because the potential energy surface must be mass-independent in the Born-Oppenheimer sense. To further simplify the 
TABLE 2: Numerical Values of the Parameters in Equation 12

\begin{tabular}{|c|c|c|c|c|c|}
\hline$C_{n}^{L, \mathrm{~S}-\mathrm{O}_{2}}$ & $C_{6}^{0}$ & $C_{6}^{2}$ & $C_{8}^{0}$ & $C_{8}^{2}$ & $C_{10}^{0} R_{\mathrm{M}} / a_{0}$ \\
\hline & 4.6016 & 4.2331 & 4.5065 & 4.1988 & 4.4397 \\
\hline$D_{\mathrm{M}} / E_{\mathrm{h}} a_{0}^{-n}$ & 61.5596 & 93.2003 & 1890.8569 & 7327.1293 & 71821.2734 \\
\hline$a_{1} / a_{0}^{-1}$ & 0.88671064 & 0.65317717 & 0.75789704 & 0.69133383 & 0.71845098 \\
\hline$a_{2} / a_{0}^{-2}$ & 0.24158216 & 0.10326597 & 0.15494697 & 0.11576995 & 0.12798057 \\
\hline$a_{3} / a_{0}^{-3}$ & 0.02034037 & -0.00017434 & 0.00553380 & -0.00015502 & 0.00025847 \\
\hline$b_{2} / a_{0}^{-2}$ & 0.05135917 & 0.13834382 & 0.06818675 & 0.16599555 & 0.06538409 \\
\hline$b_{3} / a_{0}^{-3}$ & 0.00216748 & 0.00267192 & 0.0 & 0.00424708 & 0.0 \\
\hline \multirow[t]{2}{*}{$C_{n}^{L, \mathrm{O}-\mathrm{SO}}$} & $C_{6}^{0}$ & $C_{6}^{2}$ & $C_{8}^{0}$ & $C_{8}^{2}$ & $C_{10}^{0} R_{\mathrm{M}} / a_{0}$ \\
\hline & 5.2913 & 4.7972 & 4.8684 & 4.6248 & 4.6154 \\
\hline$D_{\mathrm{M}} / E_{\mathrm{h}} a_{0}^{-n}$ & 20.9512 & 39.1726 & 1221.9609 & 5586.4509 & 64657.655 \\
\hline$a_{1} / a_{0}^{-1}$ & 0.75592241 & 0.53043052 & 0.86784761 & 0.77184416 & 0.70275904 \\
\hline$a_{2} / a_{0}^{-2}$ & 0.12843528 & 0.02416932 & 0.24924034 & 0.16413720 & 0.14028744 \\
\hline$a_{3} / a_{0}^{-3}$ & -0.00112982 & -0.01151394 & 0.02214344 & 0.00772024 & -0.00143509 \\
\hline$b_{2} / a_{0}^{-2}$ & 0.07515643 & 0.11281959 & 0.01128002 & 0.11955892 & 0.03799403 \\
\hline$b_{3} / a_{0}^{-3}$ & 0.0 & 0.0 & 0.0 & 0.0 & 0.0 \\
\hline
\end{tabular}

coordinate transformations, we introduce the following approximations:

$$
\begin{gathered}
r_{i}=\frac{A_{j} R_{j}+A_{k} R_{k}}{A_{j}+A_{k}} \\
\cos \gamma_{i}=\frac{1}{2}\left[\frac{\left(R_{k}-R_{j}\right)\left(R_{k}+R_{j}\right)}{R_{i}}+\frac{A_{k}-A_{j}}{A_{k}+A_{j}} R_{i}\right] / r_{i}
\end{gathered}
$$

These equations generalize previously reported ${ }^{47}$ expressions that have been developed for interactions involving the center of geometry of the diatomic molecule.

The atom-diatom dispersion coefficients $C_{n}^{(i)}\left(R_{i}, \gamma_{i}\right)$ of eq 7 are given by

$$
C_{n}^{(i)}\left(R_{i}, \gamma_{i}\right)=\sum_{L} C_{n}^{L}\left(R_{i}\right) P_{L}\left(\cos \gamma_{i}\right)
$$

where $P_{L}\left(\cos \gamma_{i}\right)$ are Legendre polynomials: $L=0,2, \ldots, n-$ $4(n=6,8, . ., \infty)$ and $L=1,3, \ldots, n-4(n=7,9, . ., \infty)$. Following previous work, ${ }^{53}$ the radial dependence of the dispersion coefficients has been fitted to the expression

$$
C_{n}^{L, \mathrm{~A}-\mathrm{BC}}(R)=D_{\mathrm{M}}\left[1+\sum_{i=1}^{3} a_{i} r^{i}\right] \exp \left(-\sum_{i=1}^{3} b_{i} r^{i}\right)+C_{n}^{\mathrm{AB}}+C_{n}^{\mathrm{AC}}
$$

where $r=R-R_{\mathrm{M}}, b_{1}=a_{1}$, and $R_{\mathrm{M}}$ is the internuclear distance at the maximum occurring close to the equilibrium geometry of the diatomic. The internuclear dependence of these coefficients has been calculated using a model reported elsewhere. ${ }^{53}$ Moreover, the odd- $n$ coefficients have not been considered; in fact, we have taken into account only the dispersion coefficients that are expected to contribute appreciably to the potential, namely, those whose parameters are presented in Table 2 and displayed graphically in Figure 3. In addition to the polarizability data $^{53}$ of the diatomics $\mathrm{SO}$ and $\mathrm{O}_{2}$ needed to model such coefficients, it was necessary to calculate the dipolar polarizability of the SO united atom, which is the chromium atom in the excited ${ }^{3} \mathrm{P}$ state. Using Bauschlicher ANO basis sets, ${ }^{54,55}$ we have calculated the dipolar polarizability of the SO united atom at the MRCI level using MOLPRO ${ }^{42}$ after obtaining $\alpha_{0}$ $=105.5 a_{0}{ }^{3}$ and $\alpha_{2}=23.4 a_{0}{ }^{3}$. For use in the following section, we also note that the permanent electric quadrupole moment for this excited atom at the same level of theory has been found to be $Q\left({ }^{3} \mathrm{P}, M_{L}=0\right)=-0.685 e a_{0}{ }^{2}$.
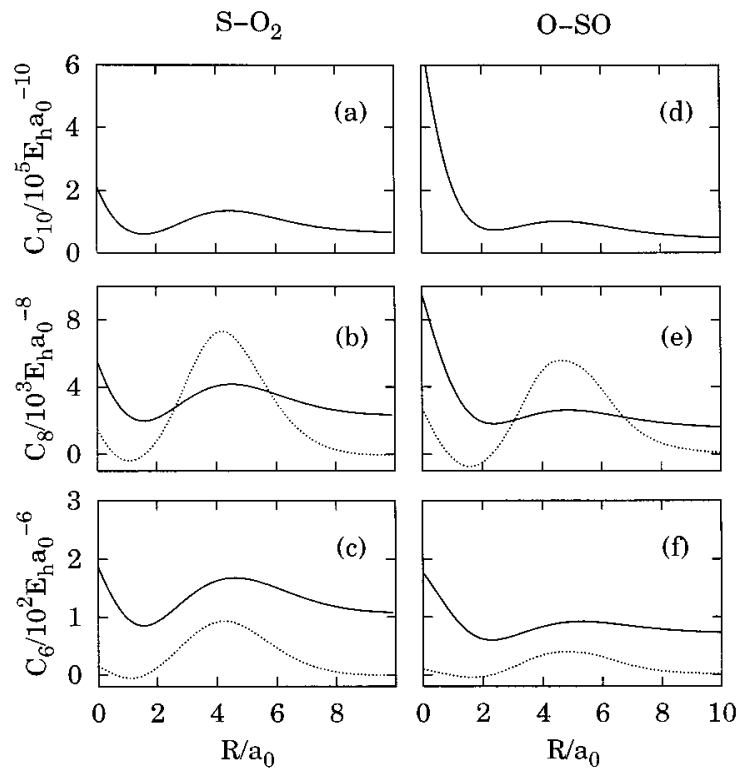

Figure 3. Dispersion coefficients for the atom-diatom asymptotic channels of $\mathrm{SO}_{2}$. (a) $C_{10}^{0}$, (b) $C_{8}^{0}$ (solid line) and $C_{8}^{2}$ (dotted line), and (c) $C_{6}^{0}$ (solid line) and $C_{6}^{2}$ (dotted line). Panels $\mathrm{d}-\mathrm{f}$ show the corresponding coefficients for channel $\mathrm{O}-\mathrm{SO}$.

3.2. Electrostatic Energy. The major long-range electrostatic potential terms of the $\mathrm{SO}_{2}$ system have their origin in the interaction of the quadrupole moment of the oxygen atom with the dipole and quadrupole moments of $\mathrm{SO}$, in addition to the interaction of the sulfur atom quadrupole with the $\mathrm{O}_{2}$ quadrupole. Similar to its treatment in eq 7, the electrostatic energy of the $\mathrm{SO}_{2}$ molecule can be approximated by

$$
\begin{aligned}
V_{\text {elec }}=\sum_{i=1}^{3} f_{i}(\mathbf{R})\left[C_{4}\left(R_{i}, r_{i}\right) \mathscr{l}_{\mathrm{DQ}}\left(\theta_{\mathrm{a}, i}, \gamma_{i}, \phi_{\mathrm{ab}, i}\right) r_{i}^{-4}+\right. \\
\left.C_{5}\left(R_{i}, r_{i}\right) \mathcal{l}_{\mathrm{QQ}}\left(\theta_{\mathrm{a}, i}, \gamma_{i}, \phi_{\mathrm{ab}, i}\right) r_{i}^{-5}\right]
\end{aligned}
$$

where $i, f_{i}(\mathbf{R}), R_{i}, r_{i}$, and $\gamma_{i}$ have the same meaning as before, $\theta_{\mathrm{a}, i}$ is the angle that defines the atomic quadrupole orientation, and $\phi_{\mathrm{ab}, i}$ is the corresponding dihedral angle. The coefficients $C_{4^{-}}$ $\left(R_{i}, r_{i}\right)$ and $C_{5}\left(R_{i}, r_{i}\right)$, say for the $i$ th channel $\mathrm{A}-\mathrm{BC}$, are given by

$$
\begin{aligned}
& C_{4}\left(R_{i}, r_{i}\right)=\frac{3}{2} Q_{\mathrm{A}} D_{\mathrm{BC}}\left(R_{i}\right) \chi_{4}\left(r_{i}\right) \\
& C_{5}\left(R_{i}, r_{i}\right)=\frac{3}{4} Q_{\mathrm{A}} Q_{\mathrm{BC}}\left(R_{i}\right) \chi_{5}\left(r_{i}\right)
\end{aligned}
$$


TABLE 3: Numerical Values of the Parameters in Equation 18

\begin{tabular}{lll}
\hline & $\mathrm{SO}\left(\mathrm{X}^{3} \Sigma^{-}\right)$ & $\mathrm{O}_{2}\left(\mathrm{X}^{3} \Sigma_{\mathrm{g}}^{-}\right)$ \\
\hline$Q_{\infty} / e a_{0}^{2}$ & 1.5003 & 0.9288 \\
$M_{6} / e a_{0}{ }^{8}$ & 9414 & 2868 \\
$R_{\mathrm{re}} / a_{0}$ & 6.060 & 3.816 \\
$A / e a_{0}^{2}$ & -0.0374939 & -0.1550177 \\
$a_{1} / a_{0}^{-1}$ & 0.7379827 & 0.4723785 \\
$a_{2} / a_{0}^{-1}$ & 0.2508889 & 1.8993563 \\
$a_{3} / a_{0}^{-3}$ & -0.0016523 & 0.1369391 \\
$b_{1} / a_{0}^{-1}$ & 2.9483605 & 1.4302665 \\
$b_{2} / a_{0}^{-2}$ & 0.6585387 & 0.6710730 \\
$b_{3} / a_{0}^{-3}$ & 0.0385221 & 0.0788006 \\
$\mathrm{rmsd} / e a_{0}{ }^{2}$ & 0.0138 & 0.0133
\end{tabular}

For convenience, these equations include the damping functions. $D_{\mathrm{BC}}\left(R_{i}\right)$ is the diatomic electric dipole moment, $Q_{\mathrm{BC}}\left(R_{i}\right)$ is the corresponding diatomic electric quadrupole moment, and $Q_{\mathrm{A}}$ is the quadrupole moment of atom A. Moreover, the angular variations of $\mathscr{C}_{\mathrm{DQ}}$ and $\mathscr{C}_{\mathrm{QQ}}$ are defined by the following: ${ }^{56}$

$$
\begin{gathered}
\mathcal{C}_{\mathrm{DQ}}\left(\theta_{\mathrm{a}}, \gamma, \phi_{\mathrm{ab}}\right)=\cos \gamma\left(3 \cos ^{2} \theta_{\mathrm{a}}-1\right)+ \\
2 \sin \theta_{\mathrm{a}} \sin \gamma \cos \theta_{\mathrm{a}} \cos \phi_{\mathrm{ab}} \\
\mathcal{\Lambda}_{\mathrm{QQ}}\left(\theta_{\mathrm{a}}, \gamma, \phi_{\mathrm{ab}}\right)=1-5 \cos ^{2} \theta_{\mathrm{a}}-5 \cos ^{2} \gamma+ \\
17 \cos ^{2} \theta_{\mathrm{a}} \cos ^{2} \gamma+2 \sin ^{2} \theta_{\mathrm{a}} \sin ^{2} \gamma \cos ^{2} \phi_{\mathrm{ab}}+ \\
16 \sin \theta_{\mathrm{a}} \sin \gamma \cos \theta_{\mathrm{a}} \cos \gamma \cos \phi_{\mathrm{ab}}
\end{gathered}
$$

Note that the dipole-quadrupole term for $\mathrm{S}-\mathrm{O}_{2}$ is absent because $\mathrm{O}_{2}$ has no dipole moment. Using the classical optimizedquadrupole (COQ) model, ${ }^{57-62}$ the angle $\theta_{\mathrm{a}}$ can be eliminated, which leads to ${ }^{62}$

$$
\begin{aligned}
& \theta_{\mathrm{a}}=\mp \arctan \left\{2 \sin \gamma\left(8 C_{5} \cos \gamma+C_{4} R\right) /\right. \\
& \left\{\left[256 C_{5}^{2} \sin ^{2} \gamma \cos ^{2} \gamma+2 C_{4} C_{5} R \cos \gamma\left(36-25 \sin ^{2} \gamma\right)+\right.\right. \\
& \left.\quad 361 C_{5}{ }^{2} \sin ^{4} \gamma+9 C_{4}{ }^{2} R^{2}+144 C_{5}{ }^{2}\right]^{1 / 2}-\left(5 C_{4}{ }^{2} R^{2}+\right. \\
& \left.\left.\left.\quad 456 C_{5}{ }^{2}\right) \sin ^{2} \gamma \mp 3 C_{4} R \cos \gamma \pm C_{5}\left(19 \sin ^{2} \gamma-12\right)+\delta\right\}\right\}
\end{aligned}
$$

$\theta_{\mathrm{a}}$ is then replaced in eqs 15 and 16 to obtain the optimum interactions according to the COQ model. A numerical parameter $\delta=10^{-10}$ was included in this expression to prevent division by zero, and the solution corresponding to the equilibrium geometry of the diatomic molecules has been used. It should also be stressed that when the dipole-quadrupole term vanishes, as for the $\mathrm{S}-\mathrm{O}_{2}$ channel, this equation simplifies to the expressions given in refs 58-60.

To model the variation of the $\mathrm{O}_{2}$ quadrupole moment with the internuclear distance, we have used the MRCI values of Lawson and Harrison; ${ }^{63}$ for SO, we have used our own FVCAS/ PT2 values calculated with the same basis set as employed for the ab initio calculations described above. These quadrupole values have been calculated as a function of the internuclear distance for the center-of-mass of the diatomic molecules and have been fitted to the following model: ${ }^{61}$

$Q(R)=A\left[1+\sum_{i=1}^{3} a_{i} r^{i}\right] \exp \left(-\sum_{i=1}^{3} b_{i} r^{i}\right)+\frac{M_{6}}{R^{6}} \chi_{8}(R)+Q_{\infty}$

$r=R-R_{\text {ref }}$ with $R_{\text {ref }}$ being a reference distance corresponding to the maximum in the $Q(R)$ curve close to the equilibrium diatomic geometry. $Q_{\infty}$ is the value of the separated-atoms quadrupole limit. The parameters so obtained are numerically defined in Table 3, while a graphical view of the fitted model
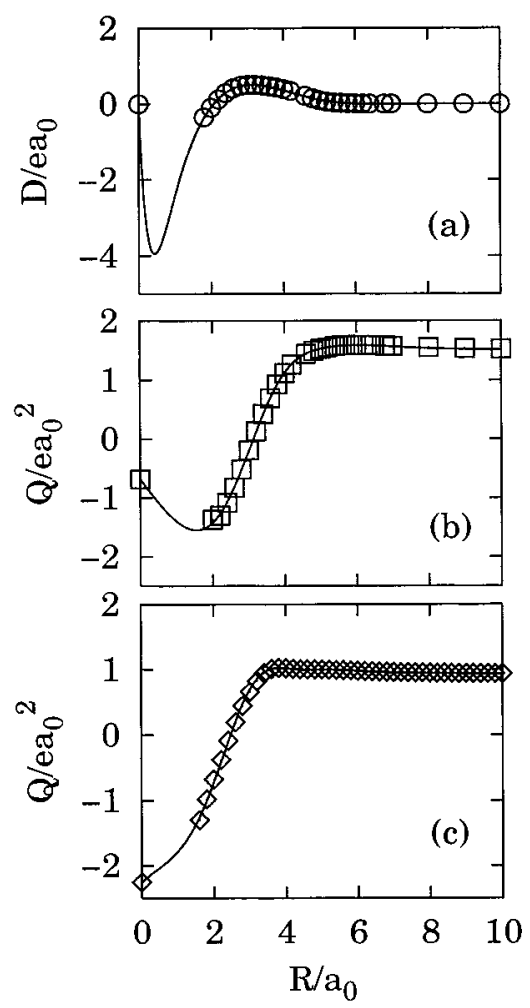

Figure 4. Electric moments as a function of interatomic distance. (a) Dipole moment of $\mathrm{SO}\left(\mathrm{X}^{3} \Sigma^{-}\right)(\mathrm{O}, \mathrm{FVCAS} / \mathrm{PT} 2$ calculation from this work); fit to eq 19, solid line. (b) Quadrupole moment of $\operatorname{SO}\left(X^{3} \Sigma^{-}\right)$ ( $\square$, FVCAS/PT2 calculation from this work). (c) $\mathrm{O}_{2}\left(\mathrm{X}^{3} \Sigma_{\mathrm{g}}^{-}\right)(\diamond$, MRCI calculations of Lawson and Harrison $\left.{ }^{63}\right)$. In panels $b$ and $c$, the fit to eq 18 is represented by the solid line.

functions can be seen in Figure 4. To fit the dipole of SO, also calculated at the same level of theory, we have used the form

$$
D(R)=A\left[1+\sum_{i=1}^{3} a_{i} r^{i}\right] \exp \left(-\sum_{i=1}^{3} b_{i} r^{i}\right)
$$

where $r=R-R_{\text {ref }}$ and $R_{\text {ref }}$ is a reference distance corresponding to the maximum in the $D(R)$ curve. The fitted coefficients have the following values: $A=0.511699 e a_{0}, R_{\text {ref }}=3.14183 a_{0}, a_{1}$ $=0.9153833 a_{0}{ }^{-1}, a_{2}=-0.1156326 a_{0}{ }^{-2}, a_{3}=-0.0972937$ $a_{0}{ }^{-3}, b_{2}=0.0269942 a_{0}{ }^{-2}$, and $b_{3}=0.0348306 a_{0}{ }^{-3}$. The rootmean-square deviation (rmsd) of the fit is $0.0137 e a_{0}$.

3.3. Three-Body EHF Energy. For each of the calculated DMBE-SEC energy points, we have calculated the three-body EHF energy by subtracting the two-body energies, the longrange three-body dispersion, and the electrostatic energies. The values so obtained have then been fitted using our previously reported distributed $n$-body polynomial ${ }^{46}$ approach. Specifically, we have used the form

$$
\begin{array}{r}
V_{\mathrm{EHF}}^{(3)}=P_{\alpha}\left(R_{1}, R_{2}, R_{3}\right) \prod_{i=1}^{3}\left\{1-\tanh \left[\gamma_{i}^{\alpha}\left(R_{i}-R_{i, \mathrm{ref}}^{\alpha}\right)\right]\right\}+ \\
P_{\beta}\left(R_{1}, R_{2}, R_{3}\right) \prod_{i=1}^{3}\left\{1-\tanh \left[\gamma_{i}^{\beta}\left(R_{i}-R_{i, \mathrm{ref}}^{\beta}\right)\right]\right\}
\end{array}
$$




$$
\begin{gathered}
P_{\alpha}\left(R_{1}, R_{2}, R_{3}\right)=c_{1}+c_{2} Q_{1}+c_{3} Q_{3}^{3}+c_{4} Q_{1}^{2}+c_{5} S_{2 \mathrm{a}}^{2}+ \\
c_{6} Q_{1} Q_{3}+c_{7} S_{2 \mathrm{~b}}^{2}+c_{8} Q_{1}^{3}+c_{9} Q_{1} S_{2 \mathrm{a}}^{2}+c_{10} S_{3}^{3}+c_{11} Q_{1}^{2} Q_{3}+ \\
c_{12} Q_{1} S_{2 \mathrm{~b}}^{2}+c_{13} Q_{3} S_{2 \mathrm{a}}^{2}+c_{14} Q_{1}^{4}+c_{15} Q_{1}^{2} S_{2 \mathrm{a}}^{2}+c_{16} S_{2 \mathrm{a}}^{4}+ \\
c_{17} Q_{1} S_{3}^{3}+c_{18} Q_{1}^{3} Q_{3}+c_{19} Q_{1}^{2} S_{2 \mathrm{~b}}^{2}+c_{20} Q_{1} Q_{3} S_{2 \mathrm{a}}^{2}+c_{21} Q_{3} S_{3}^{3}+ \\
c_{22} S_{2 \mathrm{a}}^{2} S_{2 \mathrm{~b}}^{2}+c_{23} Q_{1}^{5}+c_{24} Q_{1}^{3} S_{2 \mathrm{a}}^{2}+c_{25} Q_{1} S_{2 \mathrm{a}}^{4}+c_{26} Q_{1}^{2} S_{3}^{3}+ \\
c_{27} S_{2 \mathrm{a}}^{2} S_{3}^{3}+c_{28} Q_{1}^{4} Q_{3}+c_{29} Q_{1}^{3} S_{2 \mathrm{~b}}^{2}+c_{30} Q_{1}^{2} Q_{3} S_{2 \mathrm{a}}^{2}+ \\
c_{31} Q_{1} Q_{3} S_{3}^{3}+c_{32} Q_{1} S_{2 \mathrm{a}}^{2} S_{2 \mathrm{~b}}^{2}+c_{33} Q_{3} S_{2 \mathrm{a}}^{4}+c_{34} S_{2 \mathrm{~b}}^{2} S_{3}^{3}+c_{35} Q_{1}^{6}+ \\
c_{36} Q_{1}^{4} S_{2 \mathrm{a}}^{2}+c_{37} Q_{1}^{2} 2 S_{2 \mathrm{a}}^{4}+c_{38} Q_{1}^{3} S_{3}^{3}+c_{39} Q_{1} S_{2 \mathrm{a}}^{2} S_{3}^{3}+c_{40} S_{2 \mathrm{a}}^{6}+ \\
c_{41} S_{3}^{6}+c_{42} Q_{1}^{5} Q_{3}+c_{43} Q_{1}^{4} S_{2 \mathrm{~b}}^{2}+c_{44} Q_{1}^{3} Q_{3} S_{2 \mathrm{a}}^{2}+c_{45} Q_{1}^{2} Q_{3} S_{3}^{3}+ \\
c_{46} Q_{1}^{2} S_{2 \mathrm{a}}^{2} S_{2 \mathrm{~b}}^{2}+c_{47} Q_{1} Q_{3} S_{2 \mathrm{a}}^{4}+c_{48} Q_{1} S_{2 \mathrm{~b}}^{2} S_{3}^{3}+c_{49} Q_{3} S_{2 \mathrm{a}}^{2} S_{3}^{3}+ \\
c_{50} S_{2 \mathrm{a}}^{4} S_{2 \mathrm{~b}}^{2}
\end{gathered}
$$

is a sixth-order polynomial in symmetry coordinates and $P_{\beta^{-}}$ $\left(R_{1}, R_{2}, R_{3}\right)$ is a similar fifth-order polynomial with coefficients $c_{51}$ to $c_{84}$ (up to the term $S_{2 \mathrm{~b}}^{2} S_{3}^{3}$ ). The symmetry coordinates that have been used are defined by ${ }^{47,64}$

$$
\left[\begin{array}{l}
Q_{1} \\
Q_{2} \\
Q_{3}
\end{array}\right]=\left[\begin{array}{lll}
\sqrt{1 / 3} & \sqrt{1 / 3} & \sqrt{1 / 3} \\
\sqrt{1 / 2} & -\sqrt{1 / 2} & 0 \\
-\sqrt{1 / 6} & -\sqrt{1 / 6} & \sqrt{2 / 3}
\end{array}\right]\left[\begin{array}{l}
R_{1} \\
R_{2} \\
R_{3}
\end{array}\right]
$$

and by $S_{2 \mathrm{a}}^{2}=Q_{2}^{2}+Q_{3}^{2}, S_{2 \mathrm{~b}}^{2}=Q_{2}^{2}-Q_{3}^{2}$, and $S_{3}^{3}=Q_{3}^{3}-3 Q_{2}^{2} Q_{3}$. The complete set of parameters is constituted by $84 c_{i}$ linear coefficients, $4 \gamma_{i}^{\alpha, \beta}$ nonlinear coefficients, and 4 reference geometries $R_{i, \text { ref- }}^{\alpha, \beta}$. These coefficients have all been obtained by fitting the selected $587 \mathrm{DMBE}-\mathrm{SEC}$ points (Ab Initio Calculations section). To impose the correct long-range behavior, we have also included in the fitting procedure 855 data points generated for $r_{\{\mathrm{O}-\mathrm{SO}\}}>7.0 a_{0}$ using the sum of the two-body curves plus the dispersion and electrostatic energies. In addition, to guide the fit at short distances, we have added an extra 32 points obtained for $R_{\mathrm{AB}}<1 a_{0}$ using only the sum of the diatomic potentials. The total number of fitted points are 1474 , and the weights used for the fitting process were $w=1$ for points with $E / E_{\mathrm{h}} \geq 0, w=10^{4}$ for 21 points near the three minima, and $w=10^{2}$ for all other points. The final potential energy surface has been obtained by performing a linear leastsquares fits of the $c_{i}$ linear coefficients for fixed values of the nonlinear $\gamma_{i}^{\alpha, \beta}$ and $R_{i, \text { ref }}^{\alpha, \beta}$ coefficients. These coefficients have been optimized through a trial-and-error procedure on the condition that the final solution should lead to a potential function with no apparent spurious features. The final numerical coefficients of the three-body extended Hartree-Fock energy (eq 20) are given in Table 4. Table 5 shows the stratified rmsd of the final DMBE potential energy surface with respect to the fitted points. The data in this Table show that the final potential energy surface fits more than 1000 points covering an energy range of $200 \mathrm{kcal} \mathrm{mol}^{-1}$, with an accuracy of ca. $2 \mathrm{kcal} \mathrm{mol}^{-1}$. Note that this range of energies encompasses all the relevant features of the $\mathrm{SO}_{2}$ potential energy surface, including the energies of the ring and superoxide isomers and the $\mathrm{S}+\mathrm{O}_{2}$ and $\mathrm{O}+\mathrm{SO}$ dissociation asymptotes.

\section{Features of the Potential Energy Surface}

Table 6 reports an exhaustive list of the stationary points of the DMBE potential energy function for the title system. Such stationary points have been obtained using $2 \times 10^{4}$ random starting positions in our own implementation of a NewtonRaphson method working in projected Cartesian coordinates,
TABLE 4: Numerical Values (in Atomic Units) of the Parameters in Extended Hartree-Fock Energy Units (Equation 20)

$\begin{array}{rlrl} & \gamma_{1}^{\alpha}=\gamma_{2}^{\beta}=0.69 & & \gamma_{2}^{\alpha}=0.75 \\ & R_{1}^{\alpha}=\gamma_{2}^{\beta}=1.35 & & \gamma_{2}^{\beta}=1.00 \\ & R_{1, \text { ref }}^{\beta}=R_{2, \text { ref }}^{\beta}=2.7 & & R_{1, \text { ref }}^{\alpha}=4.6 \\ C_{1}=-377.2061509 & C_{2}=390.5876998 & C_{3}=-102.2856313 \\ C_{4}=-169.9187579 & C_{5}=32.8216007 & C_{6}=93.8827094 \\ C_{7}=48.7247862 & C_{8}=40.0386781 & C_{9}=-21.7006965 \\ C_{10}=-0.0497645 & C_{11}=-35.8932173 & C_{12}=-31.8804983 \\ C_{13}=2.5719254 & C_{14}=-5.3990814 & C_{15}=5.4569477 \\ C_{16}=0.0730623 & C_{17}=0.4352206 & C_{18}=6.9615801 \\ C_{19}=7.8199766 & C_{20}=-2.6185820 & C_{21}=-0.7976076 \\ C_{22}=-2.6955705 & C_{23}=0.3946010 & C_{24}=-0.6127501 \\ C_{25}=-0.0572129 & C_{26}=-0.1233639 & C_{27}=-0.0063605 \\ C_{28}=-0.6776244 & C_{29}=-0.8602414 & C_{30}=0.6086402 \\ C_{31}=0.2414897 & C_{32}=0.8295239 & C_{33}=0.0700467 \\ C_{34}=0.0824694 & C_{35}=-0.0121700 & C_{36}=0.0258244 \\ C_{37}=0.0049811 & C_{38}=0.0085771 & C_{39}=0.0065398 \\ C_{40}=0.0144127 & C_{41}=0.0031251 & C_{42}=0.0262868 \\ C_{43}=0.0357538 & C_{44}=-0.0404468 & C_{45}=-0.0171287 \\ C_{46}=-0.0609874 & C_{47}=-0.0161500 & C_{48}=-0.0144315 \\ C_{49}=-0.0224135 & C_{50}=-0.0152062 & C_{51}=347.3194839 \\ C_{52}=-359.7119147 & C_{53}=97.3344279 & C_{54}=150.6354161 \\ C_{55}=-26.3609221 & C_{56}=-76.6680673 & C_{57}=-44.6148061 \\ C_{58}=-31.9272711 & C_{59}=16.4699500 & C_{60}=3.2871295 \\ C_{61}=23.5367491 & C_{62}=26.8515228 & C_{63}=-0.4110505 \\ C_{64}=3.4227912 & C_{65}=-3.4187522 & C_{66}=0.4087378 \\ C_{67}=-1.3275711 & C_{68}=-3.3270241 & C_{69}=-5.4640345 \\ C_{70}=0.5890011 & C_{71}=0.7733654 & C_{72}=-0.2174864 \\ C_{73}=-0.1484332 & C_{74}=0.2320715 & C_{75}=-0.0149101 \\ C_{76}=0.1246237 & C_{77}=-0.2510945 & C_{78}=0.1825518 \\ C_{79}=0.3757480 & C_{80}=-0.0869094 & C_{81}=-0.1416933 \\ C_{82}=0.1141107 & C_{83}=0.4028050 & C_{84}=-0.1090504 \\ & & \end{array}$

TABLE 5: Stratified Root-Mean-Squar Deviations Between the Scaled Ab Initio Points and the Analytical DMBE Potential for $\mathrm{SO}_{2}$

\begin{tabular}{rcc}
\hline points & energy $\left(\mathrm{kcal} \mathrm{mol}^{-1}\right)$ & $\mathrm{rmsd}\left(\mathrm{kcal} \mathrm{mol}^{-1}\right)$ \\
\hline 25 & 20 & 1.32 \\
57 & 40 & 1.70 \\
89 & 60 & 2.08 \\
118 & 80 & 2.49 \\
157 & 100 & 2.63 \\
240 & 120 & 2.71 \\
1365 & 200 & 2.29
\end{tabular}

by which we have also calculated the harmonic frequencies at each stationary point. As can be seen from Table 6, our singlevalued potential energy surface presents a wealth of minima and saddle points, most of which were predicted from the $a b$ initio data. Obviously, very few of these points can be predicted from a potential energy surface fitted only to spectroscopic data. The properties of the global minimum(i.e., the open isomer $\mathrm{SO}_{2}$ ), namely, the geometries, energies, ${ }^{28}$ and harmonic frequencies, ${ }^{4,6}$ are well-known experimentally. It is interesting to note that the present potential energy surface, generated from average-quality $\mathrm{ab}$ initio data and scaled using the DMBE-SEC ${ }^{43}$ method, reproduces such attributes reasonably well. Also, the ring $(r-$ $\mathrm{SO}_{2}$ ) and superoxide ( $\mathrm{SOO}$ ) isomers have been studied by ab initio methods, ${ }^{22,26}$ with the latter having been observed in matrix lattices. ${ }^{65}$ Agreement with the ab initio results of Kellog et al. ${ }^{26}$ as well as with the observed frequencies in matrix support ${ }^{65}$ is fair. We have also found two van der Waals minima, $\mathrm{S} \cdots \mathrm{O}_{2}$ and $\mathrm{SO} \cdots \mathrm{O}$, that lie 8.6 and $13.9 \mathrm{kcal} \mathrm{mol}^{-1}$, respectively, below the corresponding dissociation asymptotes. All of the major features of the potential energy surface can be seen in Figures 5 and 6 , which represent an oxygen atom moving around a 
TABLE 6: Stationary Points of the DMBE Potential Energy Surface ${ }^{a}$

\begin{tabular}{|c|c|c|c|c|c|c|c|c|}
\hline & $R_{1} / a_{0}$ & $R_{2} / a_{0}$ & $R_{3} / a_{0}$ & $E / E_{\mathrm{h}}$ & $\Delta E$ & $\omega_{1}$ & $\omega_{2}$ & $\omega_{3}$ \\
\hline $\mathrm{SO}_{2}$ & $\begin{array}{l}2.696 \\
(2.704)^{b}\end{array}$ & $\begin{array}{c}2.696 \\
(2.704)^{b}\end{array}$ & $\begin{array}{c}4.579 \\
(4.667)^{b}\end{array}$ & -0.4141 & $\begin{array}{c}259.9 \\
(259.3)^{c}\end{array}$ & $\begin{array}{c}1220 \\
(1168)^{d}\end{array}$ & $\begin{array}{c}511 \\
(522)^{d}\end{array}$ & $\begin{array}{c}1285 \\
(1382)^{d}\end{array}$ \\
\hline$r-\mathrm{SO}_{2}$ & $\begin{array}{c}3.174 \\
(3.194)^{f}\end{array}$ & $\begin{array}{c}3.174 \\
(3.194)^{f}\end{array}$ & $\begin{array}{l}2.761 \\
(2.835)^{f}\end{array}$ & -0.2395 & $\begin{array}{c}109.6^{e} \\
(104.0)^{f}\end{array}$ & $\begin{array}{c}677 \\
(739)^{f}\end{array}$ & $\begin{array}{l}1032 \\
(1088)^{f}\end{array}$ & $\begin{array}{l}804 \\
(805)^{f}\end{array}$ \\
\hline SOO & $\begin{array}{c}3.081 \\
(3.079)^{f}\end{array}$ & $\begin{array}{c}4.767 \\
(4.821)^{f}\end{array}$ & $\begin{array}{c}2.461 \\
(2.483)^{f}\end{array}$ & -0.2289 & $\begin{array}{c}116.2^{e} \\
(112.8)^{f}\end{array}$ & $\begin{array}{l}1071 \\
(1384, f \\
1006)^{g}\end{array}$ & $\begin{array}{c}546 \\
(525)^{f}\end{array}$ & $\begin{array}{c}717 \\
(929, f \\
740)^{g}\end{array}$ \\
\hline $\mathrm{O} \cdots \mathrm{OS}$ & 2.782 & 6.549 & 4.788 & -0.2211 & $-13.9^{h}$ & 1137 & 166 & 345 \\
\hline $\mathrm{S} \cdots \mathrm{O}_{2}$ & 5.200 & 5.200 & 2.264 & -0.2052 & $-8.6^{i}$ & 223 & 1536 & 155 \\
\hline TS1 & 4.612 & 5.273 & 2.250 & -0.2043 & $-8.0^{i}$ & 1563 & 327 & $182 \mathrm{i}$ \\
\hline TS2 & 3.052 & 3.993 & 2.636 & -0.1964 & $-3.0^{i}$ & 1091 & $711 \mathrm{i}$ & 858 \\
\hline TS3 & 3.206 & 3.206 & 3.288 & -0.2269 & $-22.2^{i}$ & 919 & $779 \mathrm{i}$ & 507 \\
\hline TS4 & 2.840 & 5.490 & 5.461 & -0.2027 & $-2.3^{h}$ & 127 & 1048 & $322 \mathrm{i}$ \\
\hline TS5 & 2.858 & 5.224 & 3.313 & -0.2054 & $-4.0^{h}$ & 982 & 443 & $511 \mathrm{i}$ \\
\hline TS6 & $\begin{array}{c}2.763 \\
(2.731)^{j}\end{array}$ & $\begin{array}{c}2.763 \\
(2.731)^{j}\end{array}$ & $\begin{array}{c}5.526 \\
(5.462)^{j}\end{array}$ & -0.3228 & $\begin{array}{c}57.3^{e} \\
(52.1)^{j}\end{array}$ & 931 & $543 i$ & 1350 \\
\hline
\end{tabular}

${ }^{a}$ Harmonic frequencies are in $\mathrm{cm}^{-1}$, and $\Delta \mathrm{E}$ is in $\mathrm{kcal} \mathrm{mol}^{-1}$. Experimental and theoretical values from other authors are given in parentheses. ${ }^{b}$ Experimental geometry. ${ }^{68}{ }^{c}$ Experimental atomization energy. ${ }^{28}{ }^{d}$ Experimental harmonic frequencies. ${ }^{6}{ }^{e}$ Energy in reference to the open isomer $\mathrm{SO}_{2} \cdot{ }^{f} \mathrm{Ab}$ initio ${ }^{26}$ results, with energies in reference to the open isomer $\mathrm{SO}_{2} .{ }^{g}$ Observed frequencies in the matrix lattices. ${ }^{65}{ }^{h}$ Energy in reference to the channel $\mathrm{O}+\mathrm{SO},-0.1990 E_{\mathrm{h}} \cdot{ }^{i}$ Energy in reference to the channel $\mathrm{S}+\mathrm{O}_{2},-0.1916 E_{\mathrm{h}} \cdot{ }^{j}$ Energy in reference to the open isomer $\mathrm{SO}_{2} \cdot{ }^{15}$

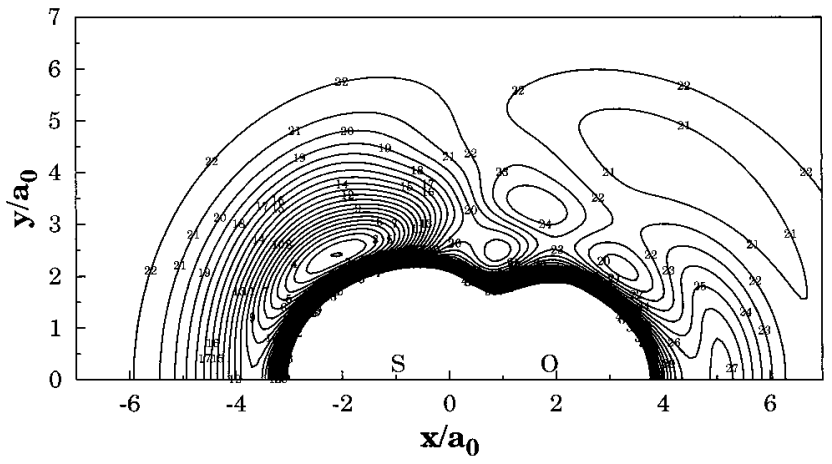

Figure 5. Contour plot of an oxygen atom moving around a partially relaxed SO diatomic. Contours start at $E_{\min }=-0.4141 E_{\mathrm{h}}$, with intervals of $\Delta E=0.01 E_{\mathrm{h}}$.

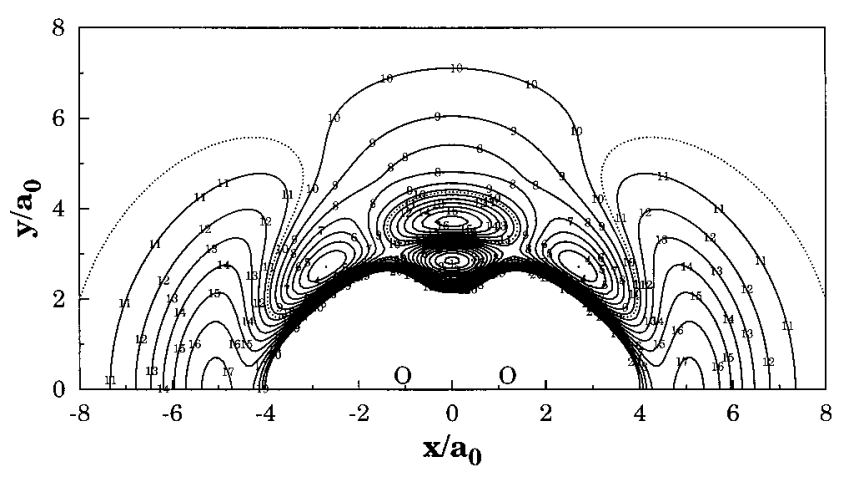

Figure 6. Contour plot for a sulfur atom moving around a partially relaxed $\mathrm{O}_{2}$ diatomic. Contours start at $E_{\min }=-0.2389 E_{\mathrm{h}}$, with intervals of $\Delta E=0.005 E_{\mathrm{h}}$. The dotted line represents the energy of dissociation to $\mathrm{S}+\mathrm{O}_{2}$.

partially relaxed SO diatomic $\left(2.6988 \leq R_{\mathrm{SO}} / a_{0} \leq 3.2289\right)$ and a sulfur atom moving around a partially relaxed $\mathrm{O}_{2}$ molecule $\left(2.1838 \leq R_{\mathrm{SO}} / a_{0} \leq 2.9839\right)$, respectively. Visible in Figure 5 are the $\mathrm{SO}_{2}, r-\mathrm{SO}_{2}$, and $\mathrm{SOO}$ minima, as well as the saddlepoints that connect them. Some of these attributes are also visible in Figure 6, namely, $r-\mathrm{SO}_{2}$, SOO isomers, and the saddle point connecting them. Another feature is the absence of a barrier for $\mathrm{S}$ approaching $\mathrm{O}_{2}$, at least for favorable atom-diatom orientations as expected from low-temperature kinetics measurements. ${ }^{66,67}$ (For clarity, the energy corresponding to separated $\mathrm{S}+\mathrm{O}_{2}$ reactants is indicated by the dotted contour.)
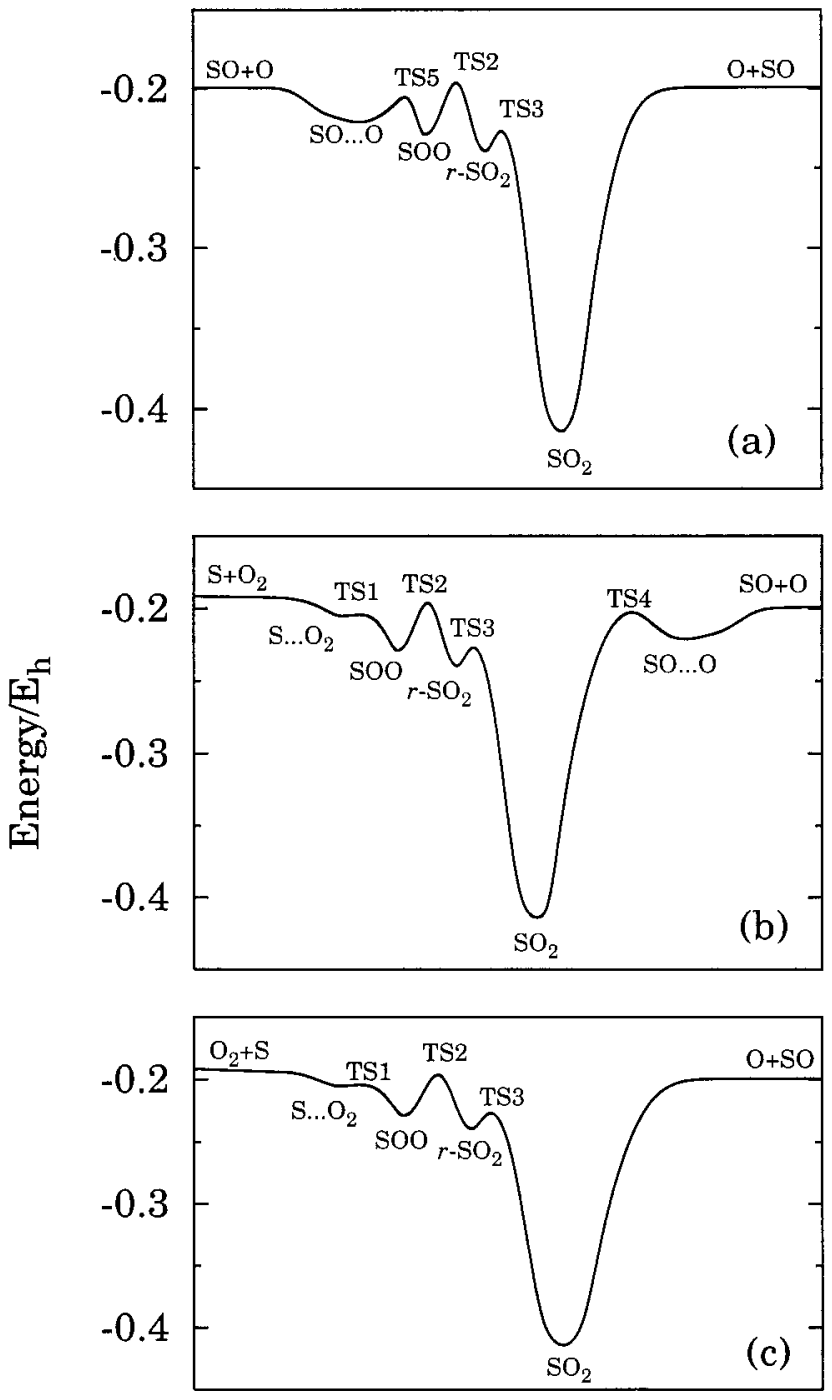

reaction coordinate

Figure 7. Minimum energy paths connecting the stationary points of the potential energy surface.

The minimum-energy paths (in the steepest-descent sense) that connect the saddle points and minima of the potential are shown in Figure 7. As usual, the asymptotic paths have been 
calculated starting with an atom that is far away from the diatomic. For $\mathrm{S}+\mathrm{O}_{2}$, all angles where the potential is attractive lead to the TS1 saddle point. Conversely, for $\mathrm{O}+\mathrm{SO}$, two possibilities can occur: for angles where the oxygen atom attacks the sulfur atom, the path leads to the global minimum; when the oxygen atom moves toward the other oxygen atom, $\mathrm{O}+\mathrm{SO}$ evolves to $\mathrm{SO} \cdots \mathrm{O}$. The other minima and saddle points are also shown in Figure 7, except for the saddle point TS6 that occurs in linear $\left(D_{\infty h}\right)$ geometries.

\section{Conclusions}

We have reported a single-valued DMBE potential energy surface for the ground electronic state of the sulfur dioxide molecule by fitting ab initio energies suitably corrected by a scaled correlation energy. An analysis of the stratified rmsd suggests that it is accurate over a range of more than $200 \mathrm{kcal}$ $\mathrm{mol}^{-1}$. Of course, given the single-valued nature of the DMBE formalism employed here, topological details such as crossing seams could not have been modeled. Despite such a limitation, the DMBE potential energy surface reported in the present work should be reliable enough to allow study of the dynamics of the reaction $\mathrm{S}+\mathrm{O}_{2} \rightarrow \mathrm{SO}+\mathrm{O}$, at least over energy ranges where the above-mentioned topological features are unimportant.

Acknowledgment. This work has the support of Fundação Para a Ciência e Tecnologia, Portugal, under Programs PRAXIS XXI and SAPIENS.

\section{References and Notes}

(1) Schofield, K. Combust. Flame 2001, 124, 137.

(2) Coudert, L.; Maki, A. G.; Olson, W. B. J. Mol. Spectrosc. 1987 124, 437.

(3) Yamanouchi, K.; Yamada, H.; Tsuchiya, S. J. Chem. Phys. 1988, $88,4664$.

(4) Yamanouchi, K.; Takeuchi, S.; Tsuchiya, S. J. Chem. Phys. 1990, $92,4044$.

(5) Lafferty, W. J.; Fraser, G. T.; Pine, A. S.; Flaud, J.-M.; CammyPeyret, C.; Dana, V.; Mandin, J.; Barbe, A.; Plateaux, J. J.; Bouazza, S. J. Mol. Spectrosc. 1992, 154, 51.

(6) Lafferty, W. J.; Pine, A. S.; Flaud, J.-M.; Cammy-Peyret, C. J. Mol. Spectrosc. 1993, 157, 499.

(7) Flaud, J.-M.; Lafferty, W. J. J. Mol. Spectrosc. 1993, 161, 396.

(8) Flaud, J.-M.; Perrin, A.; Salah, L. M.; Lafferty, W. J.; Guelachvili, G. J. Mol. Spectrosc. 1993, 160, 272.

(9) Lafferty, W. J.; Pine, A. S.; Hilpert, G.; Sams, R. L.; Flaud, J.-M. J. Mol. Spectrosc. 1996, 176, 280.

(10) Lafferty, W. J.; Flaud, J.-M.; Guelachvili, G. J. Mol. Spectrosc. 1998, $188,106$.

(11) Sako, T.; Yamanouchi, K. Chem. Phys. Lett. 1997, 264, 403.

(12) Ma, G.; Guo, H. J. Chem. Phys. 1999, 110, 8408

(13) Ma, G.; Guo, H. J. Chem. Phys. 1999, 111, 4032

(14) Xie, D.; Guo, H.; Bludsk, O.; Nachtigall, P. Chem. Phys. Lett. 2000, $329,503$. 39

(15) Zúñiga, J.; Bastida, A.; Requena, A. J. Chem. Phys. 2001, 115,

(16) Katagiri, H.; Sako, T.; Hishikawa, A.; Yazaki, T.; Onda, K.; Yamanouchi, K.; Yoshino, K. J. Mol. Struct. 1997, 413, 589.

(17) Ray, P. C.; Arendt, M. F.; Butler, L. F. J. Chem. Phys. 1998, 109, 5221.

(18) Farantos, S. C.; Murrell, J. N. Chem. Phys. 1981, 55, 205.

(19) Frederick, J. H.; McClelland, G. M.; Brumer, P. J. Chem. Phys. 1985, 83, 190 . 213.

(20) Prosmiti, R.; Farantos, S. C.; Taylor, H. S. Mol. Phys. 1994, 82,

(21) Prosmiti, R.; Farantos, S. C.; Guo, H. Chem. Phys. Lett. 1999, 311, 241

(22) Dunning, T. H., Jr.; Raffenetti, R. C. J. Phys. Chem. 1981, 85, 1350 .

(23) Jones, R. O. J. Chem. Phys. 1985, 82, 325.

(24) Bacskay, G. B.; Rendell, A. P. L.; Hush, N. S. J. Chem. Phys. 1988, 89, 5721 .
(25) Kamiya, K.; Matsui, H. Bull. Chem. Soc. Jpn. 1991, 64, 2792.

(26) Kellogg, C. B.; Schaefer, H. F., III. J. Chem. Phys. 1995, 102 4177.

(27) Pak, Y.; Woods, R. C. J. Chem. Phys. 1996, 104, 5547.

(28) Martin, J. M. L. J. Chem. Phys. 1998, 108, 2791.

(29) Bauschlicher, C. W., Jr.; Ricca, A. J. Chem. Phys. 1998, 102, 8044.

(30) Nachtigall, P.; Hrusák, J.; Bludsk, O.; Iwata, S. Chem. Phys. Lett. 1999, 303, 441 .

(31) Farantos, S. C.; Leisegang, E. C.; Murrell, J. N.; Teixeira-Dias, J. J. C.; Varandas, A. J. C. Mol. Phys. 1977, 34, 947.

(32) Carter, S.; Mills, I. M.; Murrell, J. N.; Varandas, A. J. C. Mol Phys. 1982, 45, 1053

(33) Murrell, J. N.; Craven, W.; Vincent, M.; Zhu, Z. H. Mol. Phys. 1985, 56, 839 185

(34) Bastida, A.; Zúñiga, J.; Requena, A. J. Mol. Spectrosc. 1989, 136,

(35) Kauppi, E.; Halonen, L. J. Chem. Phys. 1992, 96, 2933.

(36) Lendvay, G.; Schatz, G. C.; Harding, L. B. Faraday Discuss. 1995, 102,389

(37) Weis, B. Ph.D. Thesis, University of Frankfurt, Germany, 1991.

(38) Müller, H.; Köppel, H. Chem. Phys. 1994, 183, 107.

(39) Roos, B. O.; Andersson, K.; Fülsher, M. P.; Malmquist, P.-A.; Serrano-Andrés, L.; Pierloot, K.; Merchán, M. Adv. Chem. Phys. 1996, 93, 219.

(40) Dunning, T. H., Jr. J. Chem. Phys. 1989, 90, 1007.

(41) Kendall, R.; Dunning, T., Jr.; Harrison, R. J. Chem. Phys. 1992, 96,6769 .

(42) MOLPRO is a package of ab initio programs written by H.-J Werner and P. J. Knowles with contributions from J. Almlöf, R. D. Amos, M. J. O. Deegan, S. T. Elbert, C. Hampel, W. Meyer, K. A. Peterson, R Pitzer, A. J. Stone, P. R. Taylor, and R. Lindh, 1998.

(43) Varandas, A. J. C. J. Chem. Phys. 1989, 90, 4379.

(44) Varandas, A. J. C.; Silva, J. D. J. Chem. Soc., Faraday Trans. 1992, 88,941 .

(45) Varandas, A. J. C.; Voronin, A. I. Mol. Phys. 1995, 95, 497.

(46) Martínez-Núñez, E.; Varandas, A. J. C. J. Phys. Chem. A 2001, 105,5923

(47) Varandas, A. J. C. Adv. Chem. Phys. 1988, 74, 255.

(48) Varandas, A. J. C. In Reaction and Molecular Dynamics; Laganá, A., Riganelli, A., Eds.; Lecture Notes in Chemistry; Springer-Verlag: Berlin, 2000; Vol. 75, p 33.

(49) Caridade, P. J. S. B.; Varandas, A. J. C. Unpublished work.

(50) Varandas, A. J. C. J. Chem. Phys. 1996, 105, 3524

(51) Varandas, A. J. C. In Dynamical Processes in Molecular Physics; Delgado-Barrio, G., Ed.; IOP Publishing: Bristol, U.K., 1993; p 3.

(52) Varandas, A. J. C. Mol. Phys. 1987, 60, 527.

(53) Varandas, A. J. C.; Rodrigues, S. P. J. Chem. Phys. Lett. 1995, 245,66

(54) Bauschlicher, C. W., Jr. Theor. Chim. Acta 1995, 92, 183.

(55) Basis sets were obtained from the Extensible Computational Chemistry Environment Basis Set Database, as developed and distributed by the Molecular Science Computing Facility, Environmental and Molecular Sciences Laboratory, which is part of the Pacific Northwest Laboratory, P.O. Box 999, Richland, WA 99352, and funded by the U.S. Department of Energy. The Pacific Northwest Laboratory is a multiprogram laboratory operated by Battelle Memorial Institute for the U.S. Department of Energy under contract DE-AC06-76RLO 1830. Contact David Feller or Karen Schuchardt for further information.

(56) Buckingham, A. D. Adv. Chem. Phys. 1967, 12, 107.

(57) Varandas, A. J. C.; Brandão, J.; Quintales, L. A. M. J. Phys. Chem. 1988, 92, 3732

(58) Varandas, A. J. C. THEOCHEM (J. Mol. Struct.) 1988, 166, 59

(59) Varandas, A. J. C.; Pais, A. A. C. C. Mol. Phys. 1988, 65, 843. 9647.

60) Varandas, A. J. C.; Rodrigues, S. P. J. J. Chem. Phys. 1997, 106

(61) Rodrigues, S. P. J.; Varandas, A. J. C. Phys. Chem. Chem. Phys 2000, 2, 435 .

(62) Rodrigues, S. P. J.; Varandas, A. J. C. Unpublished work.

(63) Lawson, D. B.; Harrison, J. F. J. Phys. Chem. 1997, 101, 4781.

(64) Pastrana, M. R.; Quintales, L. A. M.; Brandão, J.; Varandas, A. J. C. J. Phys. Chem. 1990, 94, 8073 .

(65) Chen, L.; Lee, C.; Lee, Y. J. Chem. Phys. 1996, 105, 9454.

(66) Schofield, K. J. Phys. Chem. Ref. Data 1973, 2, 25.

(67) Clyne, M. A. A.; Whitefield, P. D. J. Chem. Soc., Faraday Trans. 2 1979, $75,1327$.

(68) Morino, Y.; Kikuchi, Y.; Saito, S.; Hirota, E. J. Mol. Spectrosc. 1964, 13, 95 . 